\title{
Population Pharmacokinetic and Pharmacodynamic Analysis To Evaluate a Switch to Doravirine/Lamivudine/Tenofovir Disoproxil Fumarate in People Living with HIV-1
}

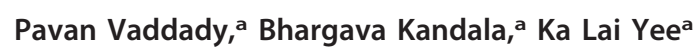 \\ aMerck \& Co., Inc., Kenilworth, New Jersey, USA
}

\begin{abstract}
Doravirine is a non-nucleoside reverse transcriptase inhibitor for treatment of human immunodeficiency virus type 1 (HIV-1) infection. A population pharmacokinetic (PK) model for treatment-naive participants in doravirine clinical studies was updated with data from switch participants in the DRIVE-SHIFT trial and used to estimate individual post hoc PK parameter values and evaluate the efficacy exposure-response relationship. The results support the 100-mg dose for people living with HIV switching to a doravirine-based regimen (This study has been registered at ClinicalTrials.gov under ClinicalTrials registration no. NCT02397096.)
\end{abstract}

KEYWORDS doravirine, fixed-dose combination, non-nucleoside reverse transcriptase inhibitor, population pharmacokinetic model, switch treatment

P eople living with HIV require lifelong antiretroviral (ARV) treatment and may switch between several ARV regimens due to poor adherence or adverse events $(1,2)$. Doravirine (DOR) is a non-nucleoside reverse transcriptase inhibitor (NNRTI) available as single entity (3) and as a fixed-dose combination with nucleoside/nucleotide reverse transcriptase inhibitors (NRTIs) lamivudine (3TC) and tenofovir disoproxil fumarate (TDF). DOR/3TC/TDF is approved for the treatment of HIV-1 infection in adults who have not received prior ARV treatment or who are virologically suppressed on a stable ARV regimen that can be appropriately replaced by DOR/3TC/TDF (4). DOR is generally well tolerated in humans, with no relevant drug-related adverse events (5-8).

DRIVE-SHIFT (7) (protocol MK-1439A-024; ClinicalTrials registration no. NCT02397096), a phase 3, open-label, randomized, active-controlled, noninferiority trial in virologically suppressed participants with HIV-1, evaluated a switch from a stable regimen of two NRTIs plus a ritonavir- or cobicistat-boosted protease inhibitor, cobicistat-boosted elvitegravir, or NNRTI to DOR/3TC/TDF. Participants were randomized (2:1) to switch to DOR/3TC/TDF on day 1 (immediate switch group [ISG]) or to continue their baseline regimen until week 24 and then switch to DOR/3TC/TDF (delayed switch group [DSG]).

The primary endpoint in DRIVE-SHIFT was the proportion of participants with an HIV-1 RNA level of $<50$ copies/ml (U.S. Food and Drug Administration [FDA] snapshot approach), with the primary comparison between ISG at week 48 and DSG at week 24. The main objectives of the current analysis were to evaluate the consistency of DOR pharmacokinetics (PK) in the ISG population with that of the treatment-naive population described previously (9) and to evaluate the exposure-response relationship between different quantiles of DOR exposure and the primary endpoint in ISG participants to further inform on the efficacy and appropriateness of a switch to a DOR-based regimen.

A total of 670 participants on stable ARV regimens were recruited to the DRIVESHIFT trial (ISG, $N=447$; DSG, $N=223$ ) (7). DOR PK samples were collected from all participants on day 1 (predose) and week 48 (predose and within 0.5 to $2 \mathrm{~h}$ postdose).
Citation Vaddady P, Kandala B, Yee KL. 2020. Population pharmacokinetic and pharmacodynamic analysis to evaluate a switch to doravirine/lamivudine/tenofovir disoproxil fumarate in people living with HIV-1. Antimicrob Agents Chemother 64:e00590-20. https://doi.org/10.1128/AAC.00590-20. Copyright $\odot 2020$ Vaddady et al. This is an open-access article distributed under the terms of the Creative Commons Attribution 4.0 International license.

Address correspondence to Pavan Vaddady, pavan.vaddady@merck.com

Received 30 March 2020 Returned for modification 15 May 2020 Accepted 13 August 2020

Accepted manuscript posted online 31 August 2020

Published 20 October 2020 
TABLE 1 Summary statistics of DOR steady-state $A U C_{0-24}, C_{\text {max }}$ and $C_{24}$ following administration of once-daily $100 \mathrm{mg}$ DOR in treatment-naive study participants and participants randomized to the ISG in the DRIVE-SHIFT trial (P024) ${ }^{a}$

\begin{tabular}{|c|c|c|c|c|c|}
\hline Phase 3 study population & $\begin{array}{l}\text { Dose } \\
\text { (mg) }\end{array}$ & Parameter & $N$ & $\begin{array}{l}\text { Geometric } \\
\text { mean }\end{array}$ & $\begin{array}{l}\text { Geometric } \\
\% \text { CV }\end{array}$ \\
\hline \multirow{3}{*}{ Treatment naive (P018 and P021) } & 100 & $\mathrm{AUC}_{0-24}(\mu \mathrm{M} \cdot \mathrm{h})$ & 730 & 38.1 & 28.8 \\
\hline & & $C_{24}(\mathrm{nM})$ & 730 & 932 & 62.7 \\
\hline & & $C_{\max }(\mathrm{nM})$ & 730 & 2,290 & 18.2 \\
\hline \multirow[t]{3}{*}{ DRIVE-SHIFT ISG (P024) } & 100 & AUC $_{0-24}(\mu \mathrm{M} \cdot \mathrm{h})$ & 443 & 41.5 & 22.8 \\
\hline & & $C_{24}(\mathrm{nM})$ & 443 & 1,110 & 36.1 \\
\hline & & $C_{\max }(\mathrm{nM})$ & 443 & 2,390 & 16.5 \\
\hline
\end{tabular}

${ }^{a} \mathrm{AUC}_{0-24}$, area under the concentration-time curve from 0 to $24 \mathrm{~h} ; \mathrm{C}_{24}$, plasma concentration $24 \mathrm{~h}$ after dose administration; $C_{\text {max }}$ maximum serum drug concentration; $C V$, coefficient of variation; DOR, doravirine; ISG, immediate switch group; $N$, number of participants.

Additional ISG PK samples were collected at weeks 4 (predose), 12 (irrespective of dosing), and 24 (predose and 0.5 to $2 \mathrm{~h}$ postdose). Only PK data from the ISG were included in the population PK analysis, given sparse sampling in the DSG (week 48 only).

To evaluate consistency of DOR PK in the phase 3 switch population with that of the phase 3 treatment-naive population, several approaches (described in Text S1 in the supplemental material) were evaluated. The exploratory analyses (approach 1) and the estimation of the combined model (approach 3b) are presented here. Observed data from DRIVE-SHIFT were initially compared with observed data from treatmentnaive participants within phase 3 trials (MK-1439-018 [P018], DRIVE-FORWARD, ClinicalTrials registration no. NCT02275780; MK-1439A-021 [P021], DRIVE-AHEAD, ClinicalTrials registration no. NCT02403674). A previously described population PK model for the treatment-naive population (9) was updated in the current analysis using DOR concentration data from the ISG of DRIVE-SHIFT. Population PK parameters, including covariates, were reestimated, and the final model was used to estimate individual post hoc PK parameter values for the switch and treatment-naive populations.

The population PK analysis data set comprised the original 341 healthy participants and 959 treatment-naive participants with HIV-1, with the addition of 443 virologically suppressed participants with HIV-1 from the DRIVE-SHIFT ISG (a total of 1,402 participants with HIV-1) (9). Four of 447 ISG individuals were excluded due to data reconciliation issues. Comparison of PK data from treatment-naive participants in prior phase 3 studies with those from DRIVE-SHIFT suggested a comparable range of DOR concentrations at different steady-state time points (see Fig. S1 in the supplemental material) and indicated the suitability of the previously developed population PK model for the DRIVE-SHIFT ISG population PK analysis (7).

The original DOR population PK model was a one-compartment model with firstorder absorption and linear apparent clearance (CL/F). Body weight and healthy versus HIV-1 infection status were covariates on apparent volume of distribution and age on apparent clearance. This model characterized the ISG data well, as supported by the diagnostic plots (see Text S1, Fig. S2, and Table S1 in the supplemental material).

The final PK parameters of the model (Table S1) were well estimated, with small standard errors. These estimates were very similar to those of the previously developed population PK model based only on data from healthy subjects and treatment-naive participants with HIV-1 (9).

PK parameters, including area under the concentration-time curve from 0 to $24 \mathrm{~h}$ $\left(\mathrm{AUC}_{\mathrm{O}-24}\right)$, maximum serum concentration $\left(C_{\max }\right)$, and plasma drug concentration $24 \mathrm{~h}$ after dose administration $\left(C_{24}\right)$ at steady state, were simulated from post hoc compartmental parameter estimates for each participant in the phase 3 studies. Table 1 and Fig. 1 show the distributions of individual steady-state $A U C_{0-24}, C_{\text {max }}$, and $C_{24}$ from the DRIVE-SHIFT ISG are comparable to those in treatment-naive phase 3 studies (P018 and P021).

The $\mathrm{AUC}_{\mathrm{O}-24}, C_{\max }$ and $C_{24}$ estimates for the DRIVE-SHIFT ISG were used in efficacy 
A

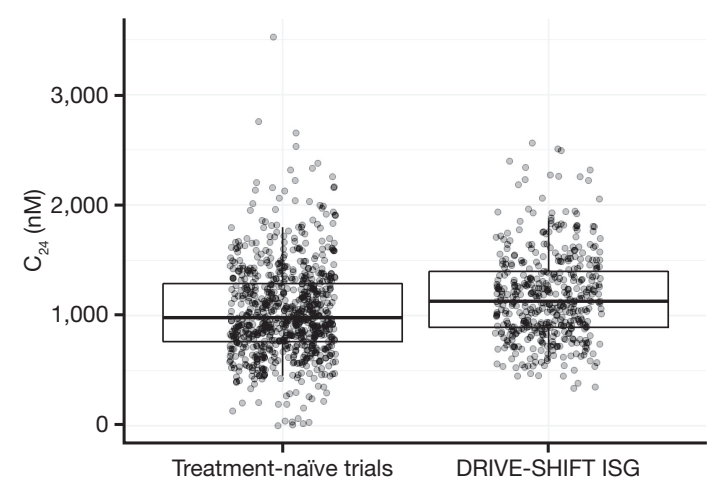

C

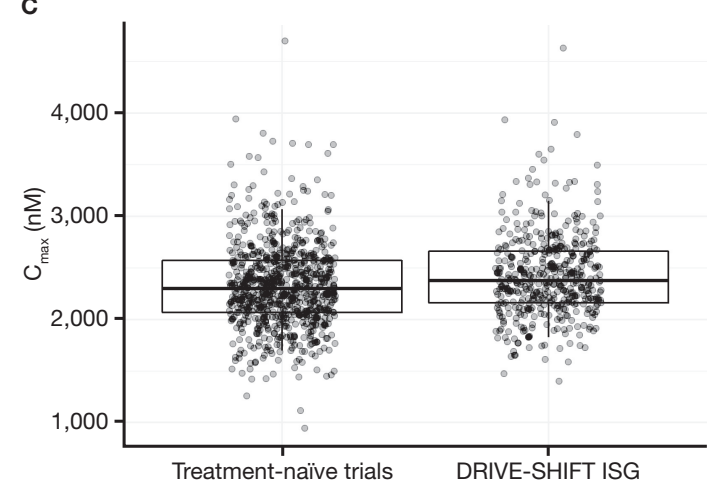

B

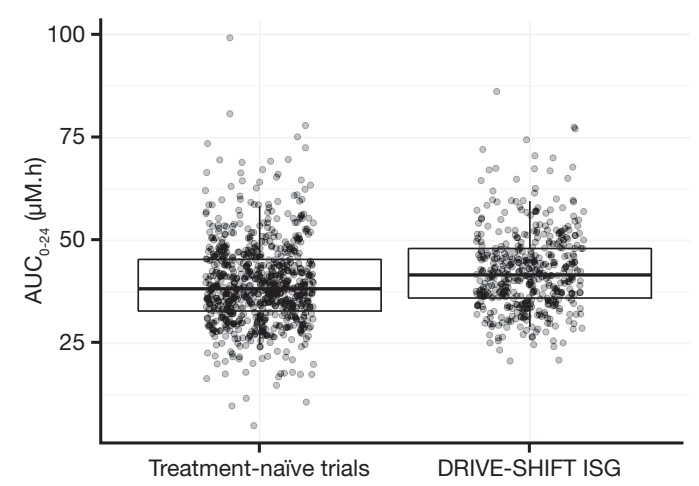

FIG 1 Comparison of steady-state DOR (A) $C_{24}$ (B) $A U C_{0-24}$, and (C) $C_{\max }$ following administration of 100 mg once-daily DOR between treatment-naive trials (P018 and P021) and participants from the ISG of DRIVE-SHIFT (P024). Boxes represent 25th, 50th, and 75th percentiles. Whiskers represent the 5th and 95th percentiles of the respective distributions for $C_{24}, A U C_{0-24},{ }_{0} C_{\text {max }}$. $\mathrm{AUC}_{0-24}$, area under the concentration-time curve from 0 to $24 \mathrm{~h} ; C_{24}$, plasma concentration $24 \mathrm{~h}$ after dose administration; $C_{\text {max }}$ maximum serum drug concentration; DOR, doravirine; ISG, immediate switch group.

exposure-response exploratory analyses for this population. The efficacy endpoints used were the proportion of ISG participants maintaining HIV-1 RNA levels of $<50$ copies $/ \mathrm{ml}$ and $<40$ copies $/ \mathrm{ml}$ at week 48 (yes/no). Analyses were conducted for (i) the primary snapshot approach specified in the phase 3 trial protocols that classified any participant with missing data as a failure and (ii) the observed failure approach, where monotone (nonintermittent) missing data for participants who discontinued treatment prematurely due to lack of efficacy were assigned as failures after discontinuation, whereas those with missing data for other reasons were excluded.

The exposure-response analysis data set included virological response data from 443 individuals. A linear exposure-effect model performed very similarly to the log exposure-effect model based on the Akaike Information Criterion values; hence the linear model was chosen for the analyses. The slope and $95 \%$ confidence interval $(\mathrm{Cl})$ of the exposure-response relationship were estimated. A $P$ value was calculated for the slope to evaluate whether it was significant (nonzero) or insignificant (not different from zero).

Slope estimates from the exposure-response analyses were not significantly different from zero ( $P$ values of $>0.05$ ), suggesting a flat exposure-response relationship with no trends between virologic response and DOR exposure over the range of exposures achieved with once-daily 100-mg doses in the DRIVE-SHIFT ISG. Consequently, structural models of increased complexity were not explored and no covariate analysis was performed. Figure 2 shows the exposure-response relationships with DOR steady-state $C_{24}$ (snapshot approach).

As DOR steady-state PK and exposure-response relationship are the same between treatment-naive and switch populations, the DOR clinical pharmacology profile char- 

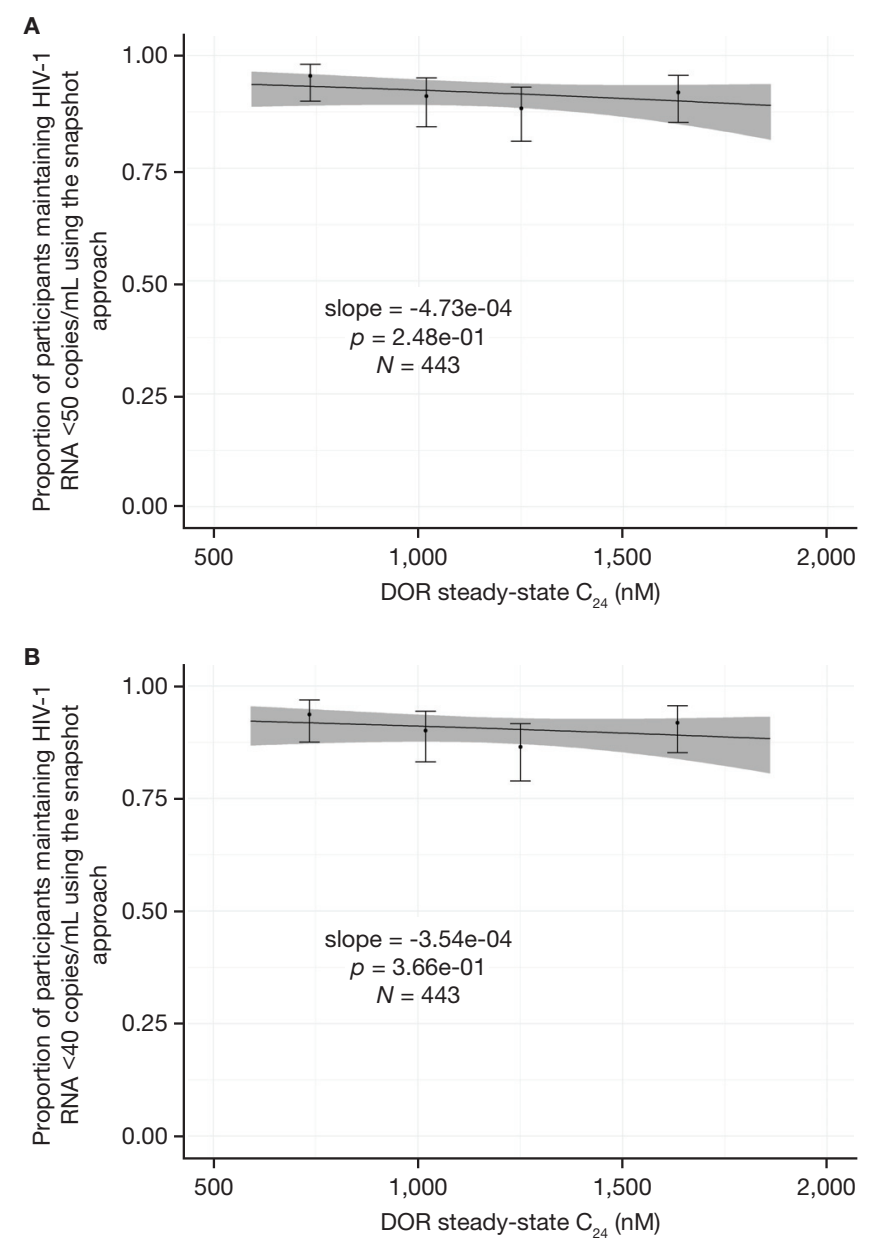

FIG 2 Predicted and observed proportion of ISG participants maintaining HIV-1 RNA at (A) $<50$ copies/ml or (B) $<40$ copies/ml using the snapshot approach as a function of DOR steady-state $C_{24}$ quartiles following administration of $100 \mathrm{mg}$ once-daily DOR $(N=443)$. Solid lines signify the mean predicted exposure-response relationship. Shaded areas represent the $95 \% \mathrm{Cl}$ of the prediction over the 5 th to 95 th percentiles of exposures. Markers and whiskers summarize the observed endpoint and $95 \% \mathrm{Cl}$ by $C_{24}$ quantile. $\mathrm{AUC}_{0-24}$, area under the concentration-time curve from 0 to $24 \mathrm{~h} ; C_{24}$, plasma concentration $24 \mathrm{~h}$ after dose administration; $C_{\text {max }}$ maximum serum drug concentration; DOR, doravirine; ISG, immediate switch group.

acterized in the treatment-naive population, including the effects of intrinsic factors and drug-drug interactions, is also applicable to the switch population. In patients switching from efavirenz, a moderate cytochrome P450 (CYP) 3A inducer, plasma concentrations of DOR may be transiently decreased as the induction effects of efavirenz are washed out $(10,11)$. However, the efficacy and PK profile of DOR in participants switching from efavirenz were found to be similar to those of participants switching from other ARV therapies (10). From a physiological perspective, sustained virologic suppression is not anticipated to impact the PK of DOR, consistent with the findings of this analysis. The similarity of DOR PK between treatment-naive and switch populations indicates that dose recommendations for DOR determined in the treatment-naive population are directly applicable to the switch population without adjustments and supports the appropriateness of the 100-mg dose of DOR within the switch population.

Data availability. The data sharing policy of Merck Sharp \& Dohme Corp., a subsidiary of Merck \& Co., Inc., Kenilworth, NJ, including restrictions, is available at http://engagezone .msd.com/ds_documentation.php. Requests for access to the clinical study data can be submitted through the EngageZone site or via email to dataaccess@merck.com. 


\section{SUPPLEMENTAL MATERIAL}

Supplemental material is available online only.

SUPPLEMENTAL FILE 1, PDF file, 0.5 MB.

\section{ACKNOWLEDGMENTS}

We thank Larissa Wenning for scientific mentorship on this project, Robert J. Valesky

for data reconciliation, and Patricia Guldin for data set programming.

Funding for this research was provided by Merck Sharp \& Dohme Corp., a subsidiary of Merck \& Co., Inc., Kenilworth, NJ. P.V., B.K., and K.L.Y. are employees of Merck Sharp \& Dohme Corp., a subsidiary of Merck \& Co., Inc., Kenilworth, NJ, USA, and may own stock and/or stock options in Merck \& Co., Inc., Kenilworth, NJ, USA.

Medical writing assistance, under the direction of the authors, was provided by Chris Whittle of CMC AFFINITY, McCann Health Medical Communications, in accordance with Good Publication Practice (GPP3) guidelines. This assistance was funded by Merck Sharp \& Dohme Corp., a subsidiary of Merck \& Co., Inc., Kenilworth, NJ, USA.

\section{REFERENCES}

1. Thompson MA, Mugavero MJ, Amico KR, Cargill VA, Chang LW, Gross R, Orrell C, Altice FL, Bangsberg DR, Bartlett JG, Beckwith CG, Dowshen N, Gordon CM, Horn T, Kumar P, Scott JD, Stirratt MJ, Remien RH, Simoni JM, Nachega JB. 2012. Guidelines for improving entry into and retention in care and antiretroviral adherence for persons with HIV: evidencebased recommendations from an International Association of Physicians in AIDS Care panel. Ann Intern Med 156:817-833. https://doi.org/10 .7326/0003-4819-156-11-201206050-00419.

2. Nachega JB, Mugavero MJ, Zeier M, Vitória M, Gallant JE. 2011. Treatment simplification in HIV-infected adults as a strategy to prevent toxicity, improve adherence, quality of life and decrease healthcare costs. Patient Prefer Adherence 5:357-367. https://doi.org/10.2147/PPA .S22771.

3. Merck Sharp \& Dohme Corp. 2019. PIFELTRO (doravirine) prescribing information. Merck \& Co, Inc, Whitehouse Station, NJ. https://www .merck.com/product/usa/pi_circulars/p/pifeltro/pifeltro_pi.pdf. Accessed 16 March 2020.

4. Merck Sharp \& Dohme Corp. 2019. DELSTRIGO (doravirine/lamivudine/ tenofovir disoproxil fumarate) prescribing information. Merck Sharp \& Dohme Corp, Whitehouse Station, NJ. https://www.merck.com/product/ usa/pi_circulars/d/delstrigo/delstrigo_pi.pdf. Accessed 16 March 2020.

5. Gatell JM, Morales-Ramirez JO, Hagins DP, Thompson M, Arasteh K, Hoffmann C, Raffi F, Osiyemi O, Dretler R, Harvey C, Xu X, Plettenberg A, Smith DE, Portilla J, Rugina S, Kumar S, Frobose C, Wan H, Rodgers A, Hwang C, Teppler H. 2019. Doravirine dose selection and 96-week safety and efficacy versus efavirenz in antiretroviral therapy-naive adults with HIV-1 infection in a Phase Ilb trial. Antivir Ther 24:425-435. https://doi .org/10.3851/IMP3323.

6. Orkin C, Squires KE, Molina JM, Sax PE, Wong WW, Sussmann O, Kaplan R, Lupinacci L, Rodgers A, Xu X, Lin G, Kumar S, Sklar P, Nguyen BY, Hanna GJ, Hwang C, Martin EA, DRIVE-AHEAD Study Group. 2019. Doravirine/ lamivudine/tenofovir disoproxil fumarate is non-inferior to efavirenz/ emtricitabine/tenofovir disoproxil fumarate in treatment-naive adults with human immunodeficiency virus-1 infection: week 48 results of the DRIVE-AHEAD trial. Clin Infect Dis 68:535-544. https://doi.org/10.1093/ cid/ciy540.

7. Johnson $M$, Kumar $P$, Molina J-M, Rizzardini $G$, Cahn $P$, Bickel $M$, Mallolas J, Zhou Y, Morais C, Kumar S, Sklar P, Hanna GJ, Hwang C, Greaves W, DRIVE-SHIFT Study Group. 2019. Switching to doravirine/ lamivudine/tenofovir disoproxil fumarate (DOR/3TC/TDF) maintains HIV-1 virologic suppression through 48 weeks: results of the DRIVESHIFT trial. J Acquir Immune Defic Syndr 81:463-472. https://doi.org/ 10.1097/QAI.0000000000002056.

8. Molina J-M, Squires K, Sax PE, Cahn P, Lombaard J, DeJesus E, Lai M-T, Xu X, Rodgers A, Lupinacci L, Kumar S, Sklar P, Nguyen B-Y, Hanna GJ, Hwang C, for the DRIVE-FORWARD Study Group. 2018. Doravirine versus ritonavir-boosted darunavir in antiretroviral-naive adults with HIV-1 (DRIVE-FORWARD): 48-week results of a randomised, double-blind, phase 3, non-inferiority trial. Lancet HIV 5:e211-e220. https://doi.org/10 .1016/S2352-3018(18)30021-3.

9. Yee KL, Ouerdani A, Claussen A, de Greef R, Wenning L. 2019. Population pharmacokinetics of doravirine and exposure-response in individuals with HIV-1. Antimicrob Agents Chemother 63:e02502-18. https://doi.org/ 10.1128/AAC.02502-18.

10. Greaves W, Wan H, Yee KL, Kandala B, Vaddady P, Hwang C. 2019. Doravirine exposure and HIV-1 suppression after switching from an efavirenz-based regimen to doravirine/lamivudine/tenofovir disoproxil fumarate. Antimicrob Agents Chemother 63:e1298-19. https://doi.org/ 10.1128/AAC.01298-19.

11. Yee KL, Sanchez RI, Auger P, Liu R, Fan L, Triantafyllou I, Lai MT, Di Spirito M, Iwamoto M, Khalilieh SG. 2017. Evaluation of doravirine pharmacokinetics when switching from efavirenz to doravirine in healthy subjects. Antimicrob Agents Chemother 61:e01757-16. https://doi.org/10.1128/ AAC.01757-16. 